\title{
EDONIO: Extended Distributed Object Network I/O Library
}

\author{
E. F. D'Azevedo \\ C. H. Romine
}


This report has been reproduced directly from the best available copy.

Available to DOE and DOE contractors from the Office of Scientific and Technical Information, P.O. Box 62, Oak Ridge, TN 37831; prices available from (615) 576-8401, FTS 626-8401.

Available to the public from the National Technical Information Service, U.S. Department of Commerce, 5285 Port Royal Rd., Springfield, VA 22161.

This report was prepared as an account of work sponsored by an agency of the Uirited States Government. Neither the United States Government nor any agency thereot, nor any of their employees, makes any warranty, express or implied, or assumes any legal liability or responsibility for the accuracy, completeness, or usefulness of any information, apparatus, product, or process disclosed, or represents that its use would not infringe privately owned rights. Reference herein to any specific commercial product, process, or service by trade name, trademark, manufacturer, or otherwise, does not necessarily constitute or imply its endorsement, recommendation, or favoring by the United States Government or any agency thereof. The views and opinions of authors expressed herein do not necessarily state or reflect those of the United States Government or any agency thereof. 


\section{DISCLAIMER}

Portions of this document may be illegible in electronic image products. Images are produced from the best available original document. 
Computer Science and Mathematics Division

Mathematical Sciences Section

\title{
EDONIO: EXTENDED DISTRIBUTED OBJECT NETWORK I/O LIBRARY
}

\author{
E.F. D'Azevedo \\ C.H. Romine
}

Mathematical Sciences Section

Oak Ridge National Laboratory

P.O. Box 2008, Bldg. 6012

Oak Ridge, TN 37831-6367

Date Published: March 1995

Research supported by the Applied Mathematical Sciences subprogram of the Office of Energy Research, U.S. Department of Energy

\author{
Prepared by the \\ Oak Ridge National Laboratory \\ Oak Ridge, Tennessee 37831 \\ managed by \\ Martin Marietta Energy Systems, Inc. \\ for the \\ U.S. DEPARTMENT OF ENERGY \\ under Contract No. DE-AC05-84OR21400
}





\section{Contents}

1 Introduction .......................... 1

2 Extended Distributed Object Network I/O Library . . . . . . . . . . . 1

3 User Interface . . . . . . . . . . . . . . . . . . . 3

4 Implementation Details . . . . . . . . . . . . . . . 18

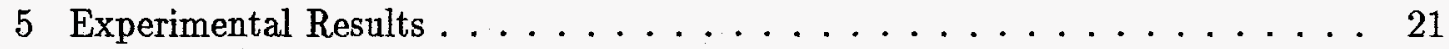

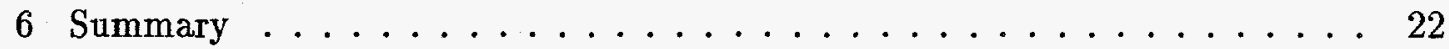

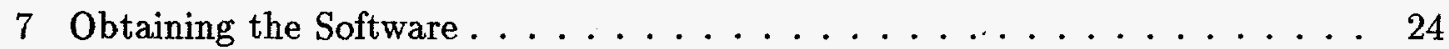

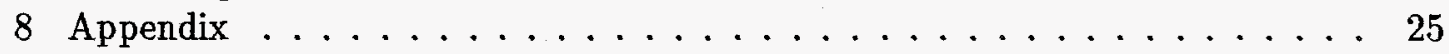

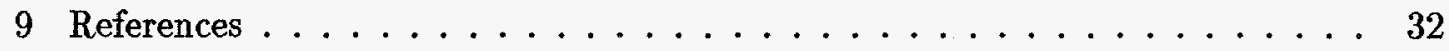





\title{
EDONIO: EXTENDED DISTRIBUTED OBJECT NETWORK I/O LIBRARY
}

\author{
E.F. D'Azevedo \\ C.H. Romine
}

\begin{abstract}
This report describes EDowIo (Extended Distributed Object Network I/O), an enhanced version of DorIo (Distributed Object Network I/O Library) optimized for the Intel Paragon Systems using the new M_ASYWC access mode. DONIO provided fast file I/O capabilities in the Intel $\mathrm{PSSC} / 860$ and Paragon distributed memory parallel environments by caching a copy of the entire file in memory distributed across all processors. EDONIO is more memory efficient by caching only a subset of the disk file at a time. DONIO was restricted by the high memory requirements and use of 32-bit integer indexing to handle files no larger than 2Gigabytes. EDONIO overcomes this barrier by using the extended integer library routines provided by Intel's $\mathrm{NX}$ operating system.

For certain applications, EDONIO may show a ten-fold improvement in performance over the native $\mathrm{NX} \mathrm{I} / \mathrm{O}$ routines.
\end{abstract}




\section{Introduction}

Multi-megabyte disk input/output operations are commonly a major bottleneck in large application codes on distributed-memory parallel supercomputers. Our first attempt to remove this bottleneck produced DONIO [2], a library of routines to provide fast parallel file I/O capabilities on Intel iPSC/860 and Intel Paragon supercomputers. DONIO caches the entire disk file across the aggregate memory of the multiprocessor in shared memory emulated by DOLIB (Distributed Object Library). This approach imposed a high memory overhead, and the use of 32-bit integer indexing restricted access to files of at most 2 Gigabytes. The new EDONIO library reduces memory overhead and provides fast $\mathrm{I} / \mathrm{O}$ on files of arbitrary size. EDONIO is implemented independently of the Distributed Object Library DOLIB [1] but uses similar IPX remote procedure calls to implement a large disk cache in the aggregate memory of the multiprocessor.

In contrast to DONIO where the entire file is cache in memory and actual disk $\mathrm{I} / \mathrm{O}$ was done only in three routines (do_open, dofflush and do_close), EDONIO caches only a portion of the disk file. At runtime, as the limited disk cache is filled, data are immediately written back to the disk in contiguous large blocks of optimal size (default is 64Kbytes to match the RAID striping parameter) for high I/O throughput. Similarly, data not found in the disk cache is dynamically read in large blocks.

The amount of memory dedicated to EDONIO is controlled by the user. A larger disk cache usually results in better performance; especially if sufficient memory is available to cache the entire file into memory. In this case EDONIO reverts back to the behavior of DONIO.

\section{Extended Distributed Object Network I/O Library}

EDONIO, like DONIO, is designed to speed up the $\mathrm{I} / \mathrm{O}$ for distributed-memory parallel applications where all processors open a common multi-megabyte shared file for simultaneous access. To access a shared file, each processor positions its own private copy of the file pointer with Iseek()'s to specific places in the file and then performs input/output operations. (Simultaneous output to overlapping regions in a shared file is nondeterministic; therefore, we assume that output operations do not overlap among processors). Such file access patterns are common in finite element codes that are 
based on subdomain decomposition. For example, the data for material properties or boundary conditions are commonly stored in shared files. This arrangement provides flexibility in solving the same problem with varying numbers or configurations of processors without rearranging the data files.

A disadvantage of large shared files is that the overhead induced by many processors attempting to access the disk file concurrently can be quite large. Machines like the Intel iPSC/860 and Paragon attempt to support simultaneous access through a special file system (CFS for the iPSC/860, PFS for the Paragon). Even with this support, the cost for concurrent access to the same file can significantly degrade the performance of a parallel program. It is common for file $\mathrm{I} / \mathrm{O}$ to be one of the most costly operations in a parallel application. On the Intel Paragon machines, the default M_UNIX mode corresponds to standard UNIX file sharing semantics that enforce atomic updates by serializing all requests. The new M_ASYNC file I/O mode allows multiple simultaneous read/write requests with no restrictions and dramatically reduces the cost of $I / O$ operations over the previous M_UNIX mode. EDONIO is designed to fully exploit the parallel M_ASYNC I/O mode by allowing all processors to perform non-overlapping I/O requests. Moreover, EDONIO uses the aggregate memory of the multiprocessor to implement a very large high-speed disk cache.

EDONIO is compatible with DONIO and offers a UNIX-like interface consisting of the 'C' callable primitives do_open(), do_read(), do_mrite(), do_lseek(), do_lsize(), doflush() and do_close(), which are similar to the open(), $\operatorname{cread}(), \operatorname{cwrite}()$, Iseek (), Isize(), flush() and $\operatorname{close}()$ routines provided by the Intel NX operating system. A Fortran callable interface, (e.g., DOREAD() for doread()), is also provided. Section 3 describes the use of these EDONIO primitives in more detail. Changing the names of the $\mathrm{I} / \mathrm{O}$ subroutines called in an application program from the $\mathrm{NX}$ version to the EDONIO version (leaving the parameters untouched) and then linking in the EDONIO library is generally all that is required to use the package. An important note: EDONIO operates only on UNIX binary files, which may be incompatible with Fortran unformatted fixed-size record files.

Many large-scale applications involving the simulation of time-evolving events are designed to output a "snapshot" or "checkpoint" of the current state of the simulation at regular intervals. A lengthy simulation may output tens (or even hundreds) of 


\section{$-3-$}

Gigabytes of data for later analysis. The original DONIO was incapable of handling files larger than 2Gigabytes. EDONIO overcomes this restriction, thereby providing rapid I/O capabilities on files of practically unlimited size (up to 16Terabytes).

\section{User Interface}

The following pages provide details on the syntax and behavior of each of the EDONIO primitives. These pages can be considered the manual for EDONIO. 


\section{do_check()}

do_check() checks the message queues for EDONIO or IPX requests from other processors, servicing any that are found.

\section{Synopsis}

int docheck ( )

subroutine docheck( )

\section{Discussion}

do_check() checks the calling processor's message queues for IPX requests from other processors. If none are found, do_check() returns immediately. Any queued requests are serviced before do_check() terminates. do_check() is provided to allow the user to avoid deadlock or slow servicing (starvation) of $\mathrm{I} / \mathrm{O}$ requests if a non-interrupt (polling) version of IPX is used. All EDONIO calls automatically perform a do_check () operation. However, do_check () should be called periodically by processors that are not involved in file $\mathrm{I} / \mathrm{O}$ operations for long periods of time. 
do_close() closes the file associated with the file descriptor and deallocates global shared resources. do_close() must be called to ensure that all buffered writes are saved to disk. In $\mathrm{C}$, do_close () returns 0 on success and -1 on failure. An implicit global synchronization is performed.

\section{Synopsis}

int do_close( int $f d$ )

subroutine doclose ( $f d$ )

integer $f d$

\section{Input parameters}

fd - $f d$ is the file descriptor obtained from do_open().

\section{Discussion}

do_close() deallocates the global shared resources used for caching the file data associated with the file descriptor $\mathrm{fd}$. For write-only and read-write files, do_close() first calls do_flush() to write out any cached data to the disk file before resources are deallocated. (If none of the cached pages are dirty, or if the file is read-only, no disk $\mathrm{I} / \mathrm{O}$ is performed).

Important note: Unlike the UNIX routines, no implicit do_close() calls are performed when the program terminates. Hence, if the user fails to call do_close() for a given file, any changes made to cached blocks that have not yet been flushed will be lost upon program termination! All processors must participate in the do_close() call. An implicit global synchronization is performed. 


\section{do_csize()}

do_csize() sets the sizes of the EDONIO read-only data cache and disk cache. An implicit global synchronization is performed.

\section{Synopsis}

int do_csize ( int data_size, int disk_size)

subroutine docsize (datasize, disksize)

integer datasize, disksize

\section{Input parameters}

datasize - datasize is the maximum amount of memory in KBytes to be allocated to the read-only data cache. A value of 0 is valid, and can be used to disable the read-only cache if no user files are opened with permission flag O.RDONLY.

disk_size - disk_size is the maximum amount of memory in KBytes to be allocated to the disk cache. A value of 0 results in an error.

\section{Discussion}

do_csize() determines the maximum memory usage allowed by EDONIO's readonly data cache and disk cache. Actual allocation of memory for the caches is done only as needed. Tip: The user might call vm_statistics() at runtime or use vm_stat on the service nodes to determine the amount of free memory (or free pages) available. To avoid excessive paging, parameters for do_csize() should not exceed the amount of free memory.

All processors must participate in the do_csize(). An implicit global synchronization is performed. 


\section{do_flush()}

doflush() forces EDONIO to write any "dirty" or "modified" blocks associated with the specified file to the disk. After do_flush(), the disk file and cached blocks are guaranteed to be consistent. In $\mathrm{C}$, doflush() returns 0 on success and -1 on failure. An implicit global synchronization is performed.

\section{Synopsis}

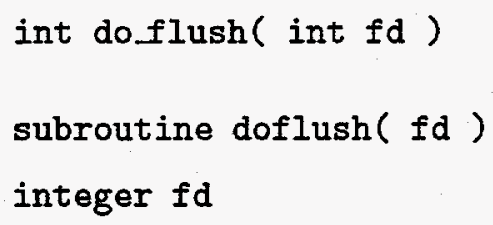

\section{Input parameters}

fd - $f d$ is the file descriptor obtained from do_open().

\section{Discussion}

doflush() forces an immediate write of any dirty blocks corresponding to the specified file to disk. If no changes have been made to the cached file since the last call to doflush(), no disk $1 / O$ will take place. doflush() is provided to support checkpointing, since in the event of a machine malfunction, all data written to the cached file will be lost. EDONIO automatically keeps track of the largest byte addressed with do_write(), so the disk file will have the correct size. However, unwritten bytes (i.e., gaps) in the file will contain garbage.

doflush() may also enhance performance of write operations. If a cache miss causes EDONIO to flush a dirty cache block, only that block is written to disk. Better I/O performance may be obtained by writing many blocks concurrently with doflush().

All processors must participate in the doflush() call. An implicit global synchronization is performed. 


\section{do_lsize(), do_esize()}

do_lsize() estimates the size of the write-only or read-write output file associated with file descriptor $\mathrm{fd}$. In $\mathrm{C}$, do_lsize() returns nbytes on success. An implicit global synchronization is performed.

\section{Synopsis}

int dolsize ( int $f d$, int nbytes )

esize_t do_esize( int $f d$, esize_t nbytes )

subroutine dolsize( $f d$, nbytes )

integer $f d$, nbytes

subroutine doesize ( $f d$, Inbytes )

integer $f d$, lnbytes(2)

\section{Input parameters}

fd - $\quad f d$ is the file descriptor obtained from do_open().

nbytes - nbytes is the estimated file size in bytes.

\section{Discussion}

do_lsize() tries to increase $I / O$ throughput by attempting to preallocate the requested disk blocks before starting write operations. Unlike DONIO it is no longer mandatory to call do_size(). Overestimation of the file size may cause overallocation and suboptimal performance, but the actual file generated on disk will be of correct (minimal) size. Calling do_lsize() for files opened for read-only access results in an error.

All processors must participate in the do_lsize(). An implicit global synchronization is performed. 


\section{do_Iseek(), do_eseek ()}

do_lseek () (do_eseek()) sets the (local) seek pointer of the open file associated with the file descriptor and returns the new seek position.

\section{Synopsis}

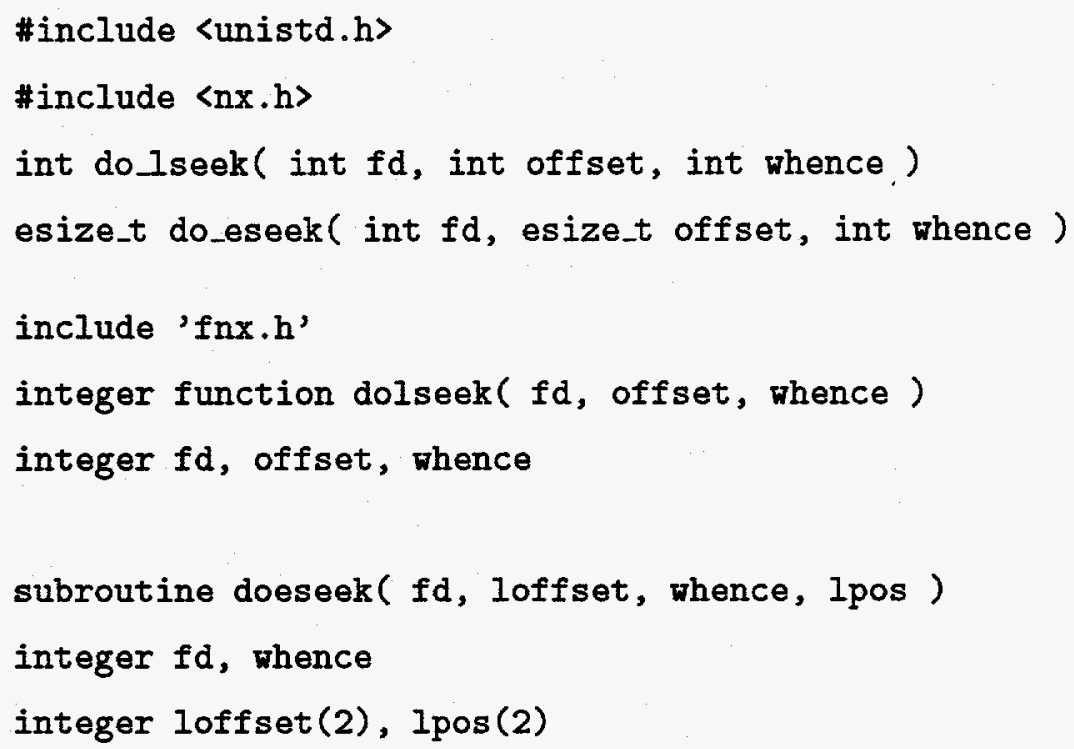

\section{Input parameters}

fd - $f d$ is the file descriptor obtained from do_open().

offset - offset is the offset in bytes. Note that EDONIO supports extended files larger than 2Gigabytes. For these extended files, the offset and returned value must be an extended integer (esize_t) in $\mathrm{C}$, or an integer array of length 2 in FORTRAN.

whence - whence determines the computation with offset. whence is one of SEEK_SET=0, SEEK_CUR=1 or SEEK_END=2. 


\section{Discussion}

do_lseek() (do_eseek()) sets the seek pointer associated with the open file specified by the descriptor $f d$ according to the value supplied for whence. whence must be one of SEEK_SET=0, SEEK_CUR=1, SEEK_END=2 defined in <unistd.h> (see $\operatorname{lseek}(2))$.

If whence is SEEKSET, the seek pointer is set to offset bytes. If whence is SEEK_CUR, the seek pointer is set to its current location plus off set. If whence is SEEK END, the seek pointer is set to the size of the file plus offset. IMPORTANT NOTE: Calling do_lseek() using whence=SEEK END is guaranteed correct only in two cases: the file must have been opened with O.RDONLY, or a call to do flush() must immediately precede the do_lseek() call. The reason is that the current file size has no meaning until all buffered writes have been flushed.

dolseek ( $f d, 0$, SEEKEND) (after doflush(), as described above) returns the size (in bytes) of the opened file associated with $\mathrm{fd}$. 


\title{
do_nio()
}

do_nio() initializes the EDONIO system. do_nio() must be called prior to opening any files with do_open(). In $\mathrm{C}$, do_nio() returns 0 on success, -1 on failure.

\section{Synopsis}

int do_nio( int myid, int nproc)

subroutine donio (myid, nproc)

integer myid, nproc

\section{Input parameters}

myid - myid is the id number of the calling processor.

nproc - nproc is the total number of processors executing.

\section{Discussion}

\begin{abstract}
All nodes must call do_nio() to initialize the EDONIO network I/O library. do_nio() sets up internal data structures and initializes the IPX subsystem. Calling do_nio() is required before any other calls to EDONIO routines. Failure to do so will result in an error.
\end{abstract}




\section{do_open()}

do_open() returns a non-negative descriptor on success. On failure, it returns -1. An implicit global synchronization is performed.

\section{Synopsis}

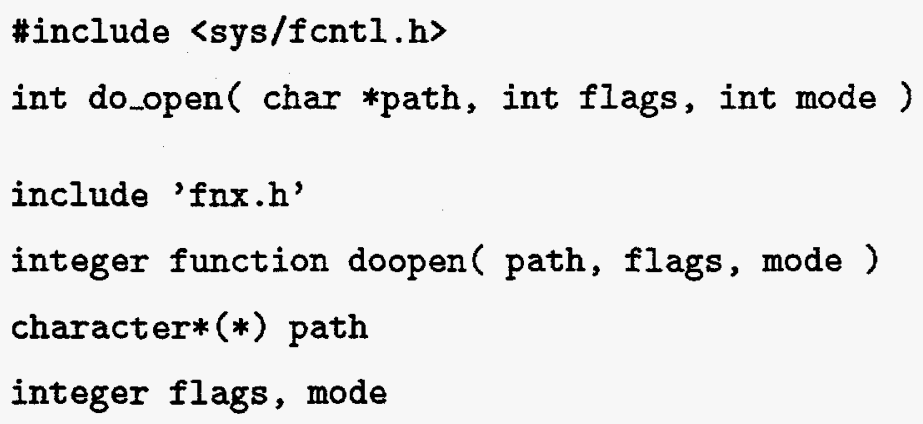

\section{Input parameters}

$$
\begin{aligned}
& \text { path - path is a null-terminated string that contains the pathname } \\
& \text { of the file. } \\
& \text { flags - flags contains the access flags. } \\
& \text { mode - mode is the file permission (see chmod(2)) used in creating } \\
& \text { the output file. mode is ignored if the file already exists. }
\end{aligned}
$$

\section{Discussion}

The routine emulates the UNIX open (see open(2) in the UNIX manual), which opens the named file specified by path for read-only, write-only or read-write access, as specified by the flags argument, and returns a descriptor for that file. For write-only or read-write access, if the file does not exist, it is created with permission mode mode (see chmod(2)). Note that do_open() differs from UNIX open if the write-only file already exists. In that case, the file is first truncated (see truncate(2)) to an empty file and then rewritten.

All processors must participate in the do_open() call. An implicit global synchronization is performed.

A Fortran example of the use of do_open() is given below: 


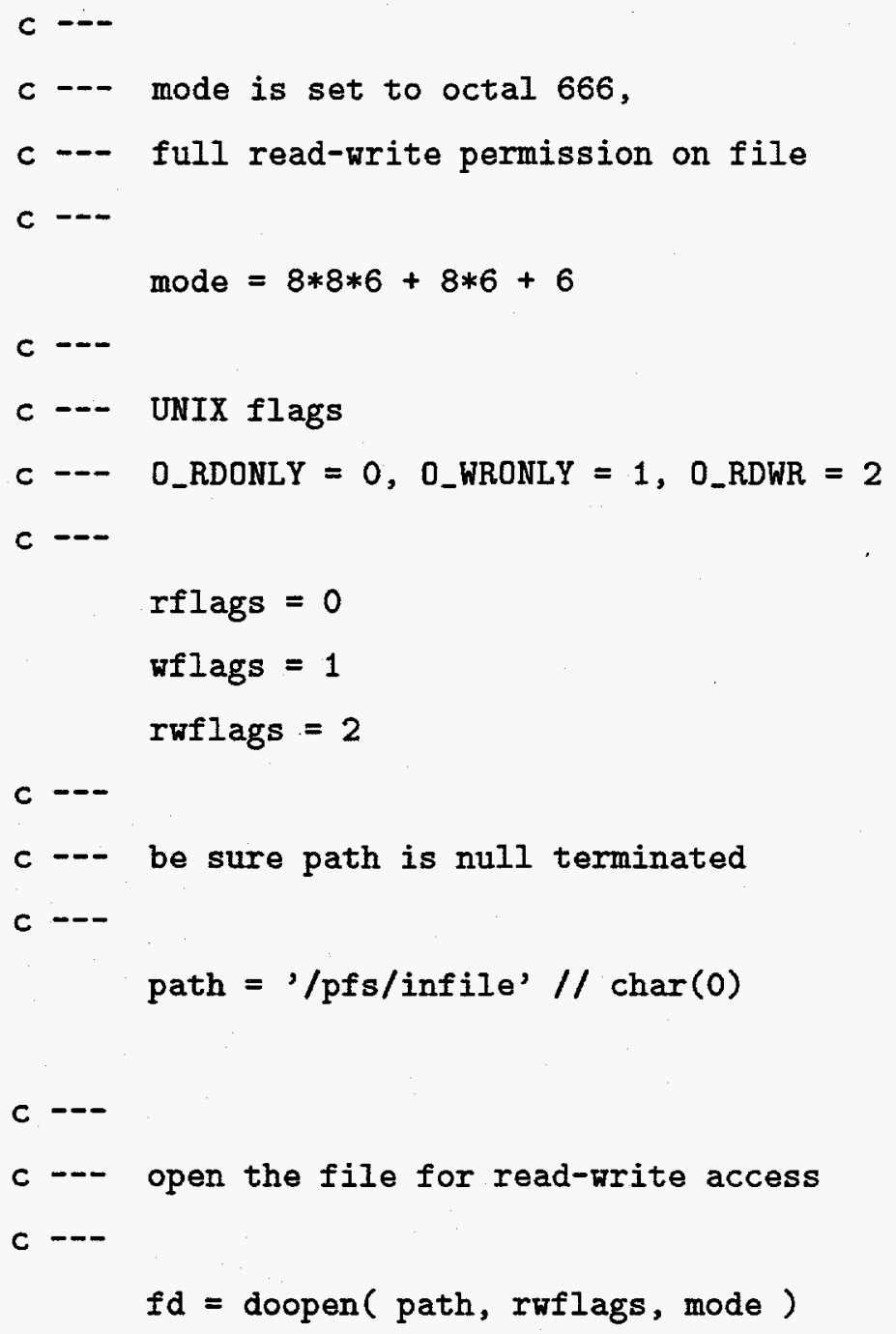




\section{do_preload()}

do_preload() fills any empty slots in the cache with blocks from the disk file, starting with the first block referenced by the minimum value of all the local seek pointers.

An implicit global synchronization is performed.

\section{Synopsis}

void do_preload( int fd )

subroutine dopreload ( fd )

integer $\mathbf{f d}$

\section{Input parameters}

$f d-f d$ is the EDONIO file descriptor for the file opened with do_open().

\section{Discussion}

do_preload() fills any empty slots in the disk cache with data from the disk. Preloading the cache is desirable when file access patterns may cause disk $\mathrm{I} / \mathrm{O}$ to be inefficient. For example, if a number of processors attempt to read common data from the same processor, then there may be significant idle time while they all wait for the data to be brought in from disk. Preloading the cache ensures that the initial disk $\mathrm{I} / \mathrm{O}$ is fully parallel and subsequent read accesses can proceed at full speed from the disk cache. Preloading starts from the point of the minimum seek location among all processors. The user can perform a do_lseek() (do_eseek()) immediately prior to the do_preload() call to ensure that the data in the cache are relevant to subsequent operations. By default, preloading starts from the beginning of file.

Note that preloading will not displace data already in the disk cache. In particular, if the cache is already full, then do_preload() has no effect. However, the user can force the creation of empty slots either by calling do_csize() to increase the memory allocated for the cache, or alternatively, the user can force a partial 
purge of the cache by using two consecutive do_csize() calls to contract and then reset the disk cache size.

All processors must participate in the do_preload() call. An implicit global synchronization is performed. 


\section{do_read()}

do_read() performs a read operation into the specified buffer. In C, do_read() returns the number of bytes read.

\section{Synopsis}

int doread ( int $f d$, void *buf, int nbytes )

subroutine doread ( $f d$, buf, nbytes )

integer $f d$, buf(*), nbytes

\section{Input parameters}

fd $\quad-f d$ is the file descriptor obtained from do_open().

buf $\quad-$ buf is the buffer.

nbytes - nbytes is the number of bytes to be read.

\section{Description}

do.read() attempts to read nbytes bytes of data from the file referenced by the descriptor fd into the buffer buf (see $\operatorname{read}(2))$.

The calling process waits (blocks) until the request is completed. Important: Note that reading past the end of file causes an error instead of partially filling the buffer. Calling doread() to read from a write-only file causes an error. The seek pointer is updated to point to the next byte in the file.

Note that the execution times for the doread() may vary substantially, depending on the access pattern and effectiveness of the disk cache. 


$$
\text { do_write(j) }
$$

do_write() performs a write operation from the specified buffer. In $\mathrm{C}$, do_write() returns the number of bytes written.

\section{Synopsis}

int do_write( int $f d$, void *buf, int nbytes )

subroutine dowrite ( $f d$, buf, nbytes )

integer $f d$, buf(*), nbytes

\section{Input parameters}

fd - $\quad f d$ is the file descriptor obtained from do_open().

buf - buf is the buffer.

nbytes - nbytes is the number of bytes to be written.

\section{Description}

do_write() attempts to write nbytes bytes of data to the file referenced by the descriptor $f d$ from the buffer buf (see write(2)).

The calling process waits (blocks) until the request is completed. Using do_write() to write to a read-only file causes an error. The seek pointer is updated to point to the next byte in the file.

Note that the execution times for do_write() may vary significantly, depending on the access pattern and effectiveness of the disk cache. 


\section{Implementation Details}

EDONIO provides a large high-speed disk cache in the aggregate memory of the Intel multiprocessor. The most important difference between EDONIO and DONIO is that the entire disk file is no longer kept in memory as in DONIO. Instead, EDONIO acts more as a true disk cache, reading and writing blocks of the file as needed. Hence EDONIO no longer requires the user to call dolsize() before do_write(). dolsize() (do_esize()) is now merely a hint to the operating system concerning the eventual file size. EDONIO automatically keeps track of the highest address actually used. If the user overestimates the file size in do_lsize() (do_esize()), then the correct (exact) size file will still be written to disk.

The conceptual view of a disk file in EDONIO is a sequence of blocks, each containing a fixed number (default 8 pages) of fixed size (default $8 \mathrm{KBytes}$ ) pages. ${ }^{1}$ Responsibility for actual disk $\mathrm{I} / \mathrm{O}$ on the blocks is assigned to the processors in a wrap-mapped fashion. Thus, in an $N$-processor configuration, processor $p$ is responsible for satisfying any $\mathrm{I} / \mathrm{O}$ requests involving blocks $p, p+N, p+2 N, \ldots$ etc.

EDONIO supplies two separate caches: the disk cache and the read-only data cache. A processor's disk cache contains blocks of the disk file that have been most recently accessed. Note that blocks are only cached in the disk cache by the processor responsible for the given block, thus eliminating concerns for cache coherency. EDONIO also provides a read-only data cache for read-only files to reduce message traffic on repeated re-reads of the same data. Read-only files cannot be updated and is completely free from cachecoherency restrictions, therefore, the read-only data cache may hold any data that has been accessed, regardless of assignment (though the actual disk read is still performed by the assigned processor).

EDONIO uses the least recently used (LRU) strategy for cache management. That is, if the cache is full when a cache miss occurs, the least recently accessed block in the disk cache is deleted to make room for the incoming cache block. For the read-only data cache, merely freeing the memory is sufficient. However, for the disk cache, the chosen block is first checked to see if it is "dirty" (i.e., has been altered). If so, it is written out to disk before it is deleted from the cache. This differs markedly from

\footnotetext{
${ }^{1}$ The xps35 Intel Paragon uses hardware pagesize of 8 Kbytes, and RAID disk stripe size is configured to be 64 Kbytes.
} 
DONI0, where the cache was set large enough to contain the entire file, thus eliminating the need for disk I/O until the file was closed.

In EDONIO, all processors must participate concurrently in do_open(), do_lsize() (do_esize()), doflush() and do_close(). The processors are synchronized when opening a shared file with do_open() so that EDONIO can set up common data structures. They are synchronized in doflush () and in do_close() to ensure that there are no outstanding read or write requests.

EDONIO must deviate from the UNIX file system with respect to file permissions. The UNIX file systems allow a user to open an existing file with flag O_WRONLY (assuming the file mode allows write access) in a directory in which the user does not have read access. EDONIO cannot allow this, since it is impossible for EDONIO to act as a disk cache on a file without read permission. For simplicity, we assume that the user has read permission on any files that will be accessed with do_open(). Moreover, although EDONIO supports a write-only file mode (as a safety check to prevent read operations on the file), the actual file permissions must allow both reading and writing.

The original DONIO did not support an APPEND mode for file I/O. Instead, the user was advised to open separate files for each logically separate set of data, largely because of the inherent limitation on file size in DONIO. With EDONIO, the UNIX O_APPEND is still not directly supported but file size is no longer a concern, as we now fully support files of practically unlimited size (up to 16Terabytes). The user can append to a file by first seeking to end of file (see description on dolseek() and doflush()) before writing.

With EDONIO, the execution times for do_read() and do_write() may vary significantly depending on the ratio of cache hits/misses. The user can reduce these times in several ways. The size of the cache can be increased (see do_csize()) to improve the probability of cache hits, or preloading of the cache (see do preload()) can also improve $1 / 0$ performance.

Consider the sequence of events initiated by a do_read() request. First, the disk blocks involved are identified. If the disk block is assigned to the same calling processor, the local disk cache is searched. A cache miss causes EDONIO to load these blocks into the local disk cache, displacing other blocks if necessary. For any blocks assigned to 
other processors, the IPX ${ }^{2}$ system [3] is used to request the data from the processors that "own" those blocks. The read request is satisfied after the remote data are received. If the file was opened as a read-only file, the incoming data are also placed in the local read-only cache, to reduce message passing traffic should the same data be referenced in subsequent read operations. Note the read-only data cache holds only remote (nonlocal) data.

A do_write() operation is similar. Again, the disk blocks to be written are identified. Blocks assigned to the same processor are loaded into the cache if they are not already there. EDONIO uses the IPX " on" routine (a type of "remote procedure call") to cause other processors to update blocks assigned to them. On the iPSC/860, IPX uses the NX hrecv() interrupt mechanism to preempt a processor to service IPX requests. However, on the Intel Paragon, hrecv() is not a true interrupt handler but spawns a separate thread that executes concurrently with the main computation. The extensive use of masktrap() for exclusive access to critical sections incurs a very high overhead on the Paragon. We have chosen to use a more efficient non-interrupt (polling) version of IPX for use on the Paragon. Because IPX requests are serviced only when the message queue is polled, and processors must supply data or update blocks at the request of other processors, the user must be careful to prevent deadlock or starvation. EDONIO provides the do_check() routine to examine the message queue for IPX requests. For example, code that uses a subset of the processors to handle all the disk $I / O$ will fail unless the remaining processors periodically call do_check (), since IPX requests to these processors will not be serviced. See the manual page for do_check () for further discussion.

We have included a subprogram for preloading the disk-cache to enhance performance of the disk I/O. Preloading of the disk-cache is particularly desirable immediately after opening an existing file, where disk $\mathrm{I} / \mathrm{O}$ during preloading proceeds in parallel. Preloading is not guaranteed to improve I/O performance since it depends on the access pattern and size of disk cache. See the manual page for do_preload() for further details.

\footnotetext{
${ }^{2}$ IPX is available by anonymous FTP from msg.das.bnl.gov under the directory /pub/ipx.
} 


\section{Experimental Results}

In this section we present a rough comparison of disk performance by EDONIO versus native NX routines. The Fortran source code is included in the Appendix. The code is a contrived example that simulates the disk $\mathrm{I} / \mathrm{O}$ common in finite element codes by performing multiple direct access 1 seek ()'s, cread()'s and cwrite()'s. This example generates the element-to-vertex list for a three dimensional nex $\times$ ney $\times$ nez grid. The elements are assumed to be ordered with $z$-index varying fastest, then $x$ then $y$. Elements along the vertical direction are grouped in buffer mibuf before writing to obtain better disk performance. Note that the element-to-vertex list file is independent of the number of processors. The same file is later read again.

Since operating system patches and compiler upgrades are regularly applied to the 512-processor xps35 Intel Paragon system at the Oak Ridge National Laboratory, and EDONIO is currently undergoing performance tuning, the performance numbers listed should be taken only as approximate and reflect only the current state of affairs (Feb 1995, OS version R1.2.5). Moreover, background disk activity by other concurrently running applications may also affect the timings. Three problems were used for testing: a small $100 \times 100 \times 100(1,000,000$ elements $)$ problem, a medium $200 \times 200 \times 200$ $(8,000,000$ elements $)$, and a large $300 \times 300 \times 300(27,000,000$ elements $)$ problem.

Table 5.1 show the effect of varying the amount of memory allocated to the disk cache in EDONIO on 22 nodes on a $200 \times 200 \times 200$ grid (file size is $256 \times 10^{6}$ bytes). We see from Table 5.1 that optimal performance is obtained when the aggregate disk cache can hold the entire file. Table 5.2 shows preloading the disk cache can reduce I/O time in read for 16 nodes on $121 \times 121 \times 91$ grid (file size is $42,634,592$ bytes). Runtimes are obtained from dclock().

Tables 5.3-5.5 list the runtimes (in seconds) for the three problems. All runs have EDONIO configured to use $512 \mathrm{Kbytes}$ for read-only data cache, $4096 \mathrm{Kbytes}$ for disk cache and with cache preloading. Note that with the default $4096 \mathrm{Kbytes}$ allocated for the disk cache, 8,62 and 206 processors are needed to hold the small, medium and large problems (respectively) in memory. The label wopen (wclose) denotes the time for opening (closing) a file for write-only access; similarly, ropen and rclose apply to read-only access. Note that read and mrite times in EDONIO decrease with the addition of more processors. As more processors are used, fewer messages per processor 
Table 5.1: Effect on disk cache size on EDONIO, all times in seconds.

\begin{tabular}{|r|r|r|r||r|r|r|r|}
\hline \hline Cache (KBytes) & wopen & write & wclose & ropen & preload & read & rclose \\
\hline \hline 1024 & 3.0 & 20.6 & 0.7 & 1.6 & 0.7 & 55.6 & 0.2 \\
2048 & 1.7 & 21.2 & 1.5 & 1.2 & 1.3 & 52.7 & 0.3 \\
4096 & 1.6 & 17.3 & 3.2 & 2.2 & 2.6 & 46.2 & 0.3 \\
8192 & 1.5 & 13.4 & 6.2 & 1.2 & 5.5 & 29.4 & 0.3 \\
12288 & 1.6 & 9.6 & 6.6 & 2.2 & 10.3 & 20.3 & 1.5 \\
\hline
\end{tabular}

Table 5.2: Effect of do_preload()on EDONIO, all times in seconds.

\begin{tabular}{|r|r|r|r||r|r|r|r|}
\hline & wopen & write & wclose & ropen & preload & read & rclose \\
\hline \hline With preload & 2.2 & 3.1 & 2.4 & 1.3 & 1.3 & 4.7 & 0.2 \\
No preload & 1.2 & 3.1 & 1.8 & 0.8 & 0.0 & 25.3 & 0.2 \\
NX & 1.4 & 38.5 & 0.2 & 0.7 & 0.0 & 25.4 & 0.2 \\
\hline
\end{tabular}

are generated. Moreover, more total aggregate memory (4Mbytes per processor) is available for the disk cache. wclose and preload involve physical disk activity to write out or read in data into the aggregate disk cache; hence as the disk cache size increases with more processors, more data are transfered and more time for disk $\mathrm{I} / \mathrm{O}$ may be required.

We see that with a large enough disk cache, EDONIO may offer nearly a ten-fold improvement over native NX routines. However, if the disk cache is too small to be effective, performance of EDONIO may be similar to native NX. EDONIO fully exploits the new M_ASYNC mode in achieving over 20Megabytes per second overall disk throughput to the /pfs. By comparison, DONIo with the default M_UNIX mode obtained only about 5 Megabytes per second disk throughput.

\section{Summary}

We have described EDONIO, a fast file I/O emulation library for the Intel iPSC and Paragon distributed memory multiprocessors. EDONIO provides an easy to use interface, and with minimal change to the source of an iPSC/860 or Paragon parallel program may improve file $\mathrm{I} / \mathrm{O}$ by a ten-fold speedup. Similar to the shared-memory library DOLIB, EDONIO uses the IPX message system to provide a very large high-speed disk 
Table 5.3: Runtimes (in seconds) of EDONIO (NX) routines on $100 \times 100 \times 100$ grid, file size is $32 \times 10^{6}$ bytes.

\begin{tabular}{|r|r|r|r||r|r|r|r|}
\hline \hline processor & wopen & write & wclose & ropen & preload & read & rclose \\
\hline \hline 1 & $1.9(1.0)$ & $29.1(293.7)$ & $0.7(0.1)$ & $0.7(0.4)$ & 2.5 & $44.9(213.7)$ & $0.1(0.1)$ \\
2 & $1.5(1.0)$ & $20.9(187.8)$ & $0.6(0.1)$ & $0.6(0.5)$ & 2.8 & $39.5(83.8)$ & $0.1(0.1)$ \\
4 & $2.0(0.9)$ & $13.2(84.2)$ & $0.7(0.1)$ & $0.6(0.6)$ & 2.8 & $22.6(48.9)$ & $0.1(0.1)$ \\
8 & $1.4(0.9)$ & $6.4(50.5)$ & $1.2(0.1)$ & $0.7(0.6)$ & 1.9 & $8.2(34.2)$ & $0.2(0.1)$ \\
16 & $1.2(1.5)$ & $3.5(26.8)$ & $1.1(0.2)$ & $1.0(0.7)$ & 1.8 & $4.3(17.0)$ & $0.3(0.2)$ \\
32 & $2.0(1.6)$ & $2.0(20.5)$ & $1.8(0.4)$ & $1.3(1.4)$ & 1.0 & $2.3(10.0)$ & $0.4(0.4)$ \\
64 & $3.1(2.8)$ & $1.3(22.6)$ & $2.4(0.7)$ & $2.5(3.0)$ & 0.9 & $1.3(9.5)$ & $0.8(0.9)$ \\
\hline
\end{tabular}

Table 5.4: Runtimes (in seconds) of EDONIO (NX) routines on $200 \times 200 \times 200$ grid, file size is $256 \times 10^{6}$ bytes.

\begin{tabular}{|r|r|r|r||r|r|r|r|}
\hline \hline processor & wopen & write & wclose & ropen & preload & read & rclose \\
\hline \hline 16 & $3.1(1.3)$ & $31.3(141.2)$ & $1.9(0.2)$ & $1.4(0.8)$ & 2.2 & $84.5(89.5)$ & $0.3(0.2)$ \\
32 & $3.1(2.1)$ & $15.5(122.4)$ & $3.2(0.4)$ & $1.5(1.3)$ & 3.6 & $30.1(49.3)$ & $0.4(0.4)$ \\
64 & $3.0(3.5)$ & $5.3(118.6)$ & $7.6(0.7)$ & $2.5(2.1)$ & 7.9 & $7.2(48.0)$ & $0.8(0.7)$ \\
128 & $4.7(4.7)$ & $3.0(89.2)$ & $10.7(1.5)$ & $4.3(3.7)$ & 7.7 & $4.0(47.5)$ & $1.6(1.4)$ \\
\hline
\end{tabular}

Table 5.5: Runtimes (in seconds) of EDONIO (NX) routines on $300 \times 300 \times 300$ grid, file size is $864 \times 10^{6}$ bytes.

\begin{tabular}{|r|r|r|r||r|r|r|r|}
\hline \hline processor & wopen & write & wclose & ropen & preload & read & rclose \\
\hline \hline 32 & $2.1(1.5)$ & $45.9(262.0)$ & $5.2(0.4)$ & $2.6(2.3)$ & 3.4 & $119.5(111.4)$ & $0.4(0.3)$ \\
64 & $2.9(2.8)$ & $24.1(218.1)$ & $7.3(0.7)$ & $2.8(2.2)$ & 6.5 & $56.7(108.8)$ & $0.8(0.7)$ \\
128 & $4.9(4.5)$ & $14.1(360.3)$ & $23.1(1.5)$ & $4.6(4.8)$ & 15.8 & $21.4(105.2)$ & $1.5(1.5)$ \\
\hline
\end{tabular}


cache in the aggregate memory of the multiprocessor. Disk I/O operations are in large blocks to fully exploit the new M_ASYNC I/O mode. EDONIO is more memory efficient than DONIO and can access files of practically unlimited size.

\section{Obtaining the Software}

To obtain the source code for EDONIO the reader should send email to the authors: e6d@ornl.gov or rominech@ornl.gov.

\section{Acknowledgments}

The authors would like to express appreciation to Bob Marr, Ron Peierls and Joe Pasciak for the IPX package, which simplified the development of EDONIO. We also thank Tom Dunigan, John Drake, David Walker and Pat Worley for suggesting improvements both to EDONIO and to this report. 


\section{Appendix}

In this appendix, we list the Fortran source code used in comparing the performance of EDONIO and NX disk operations. Note that either EDONIO or NX routines can be selected by a flag at compile time.

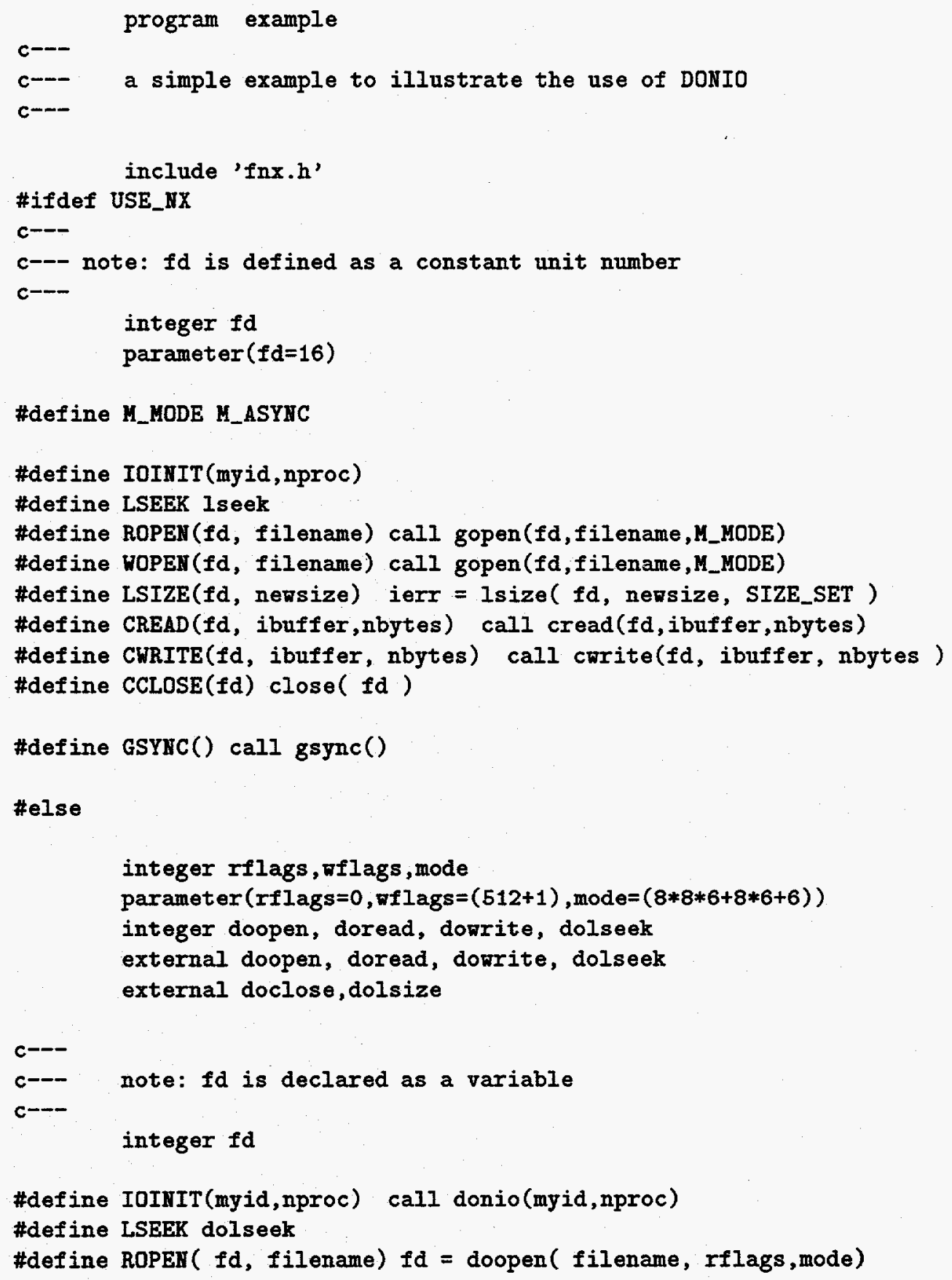




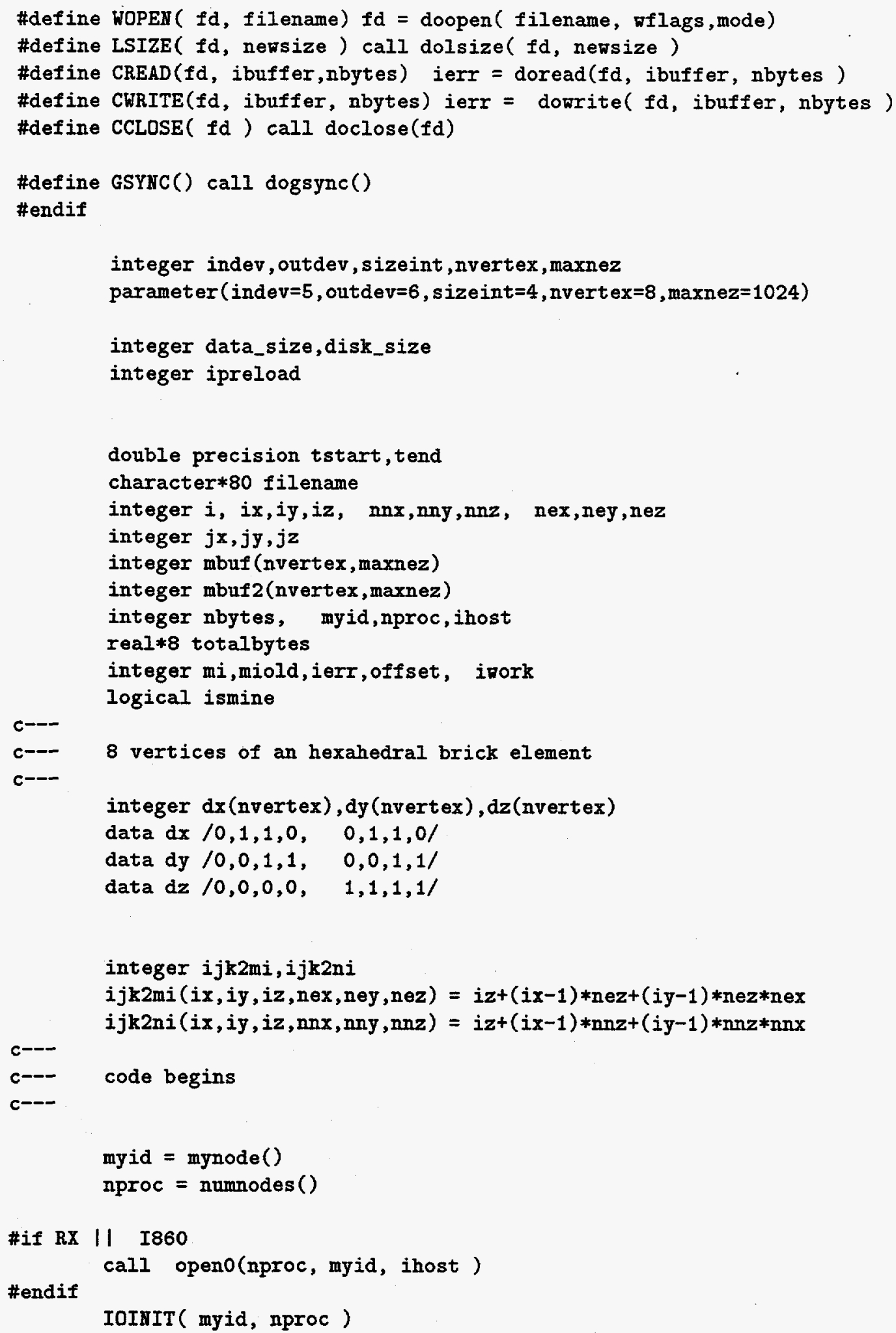




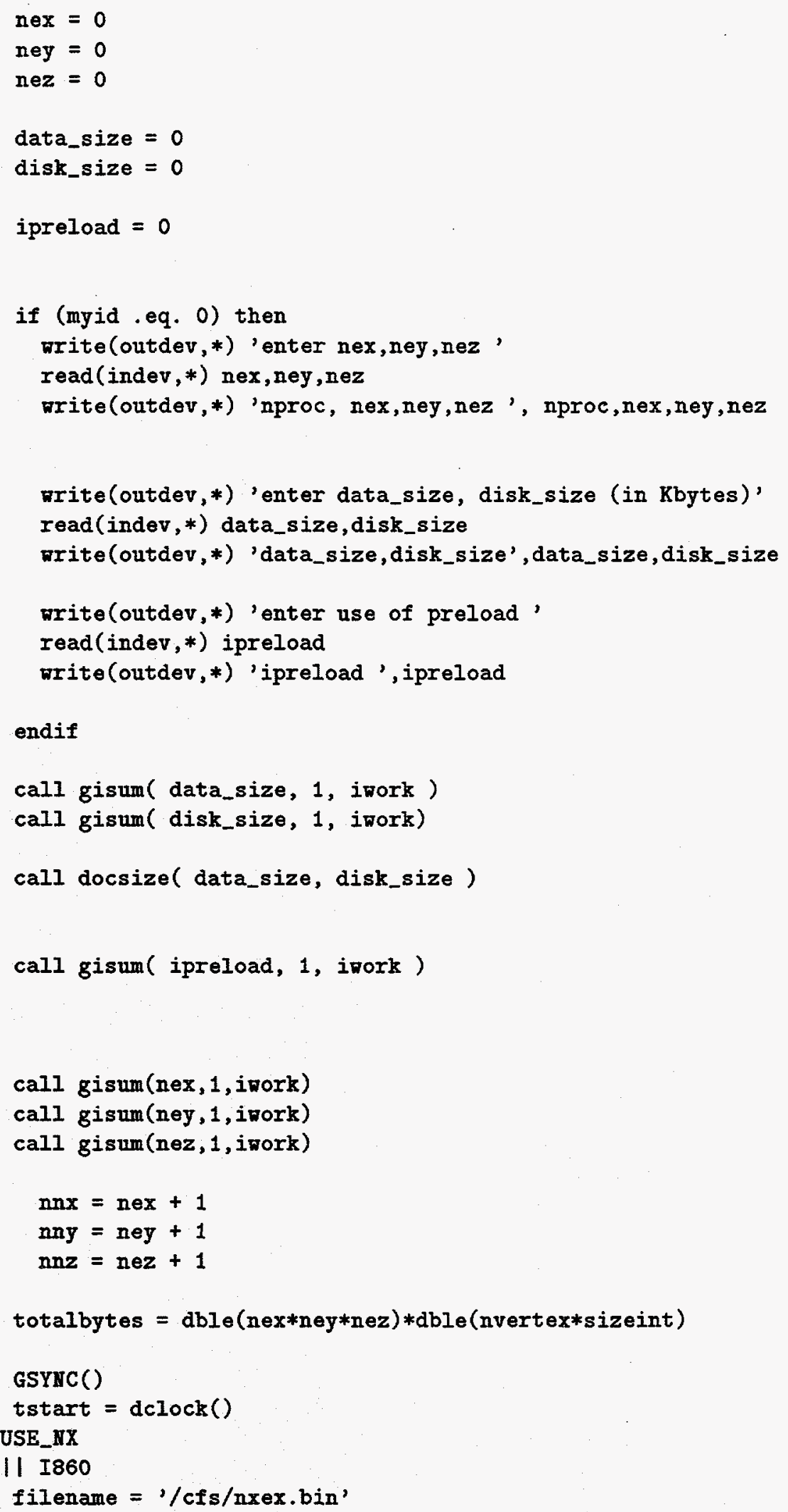




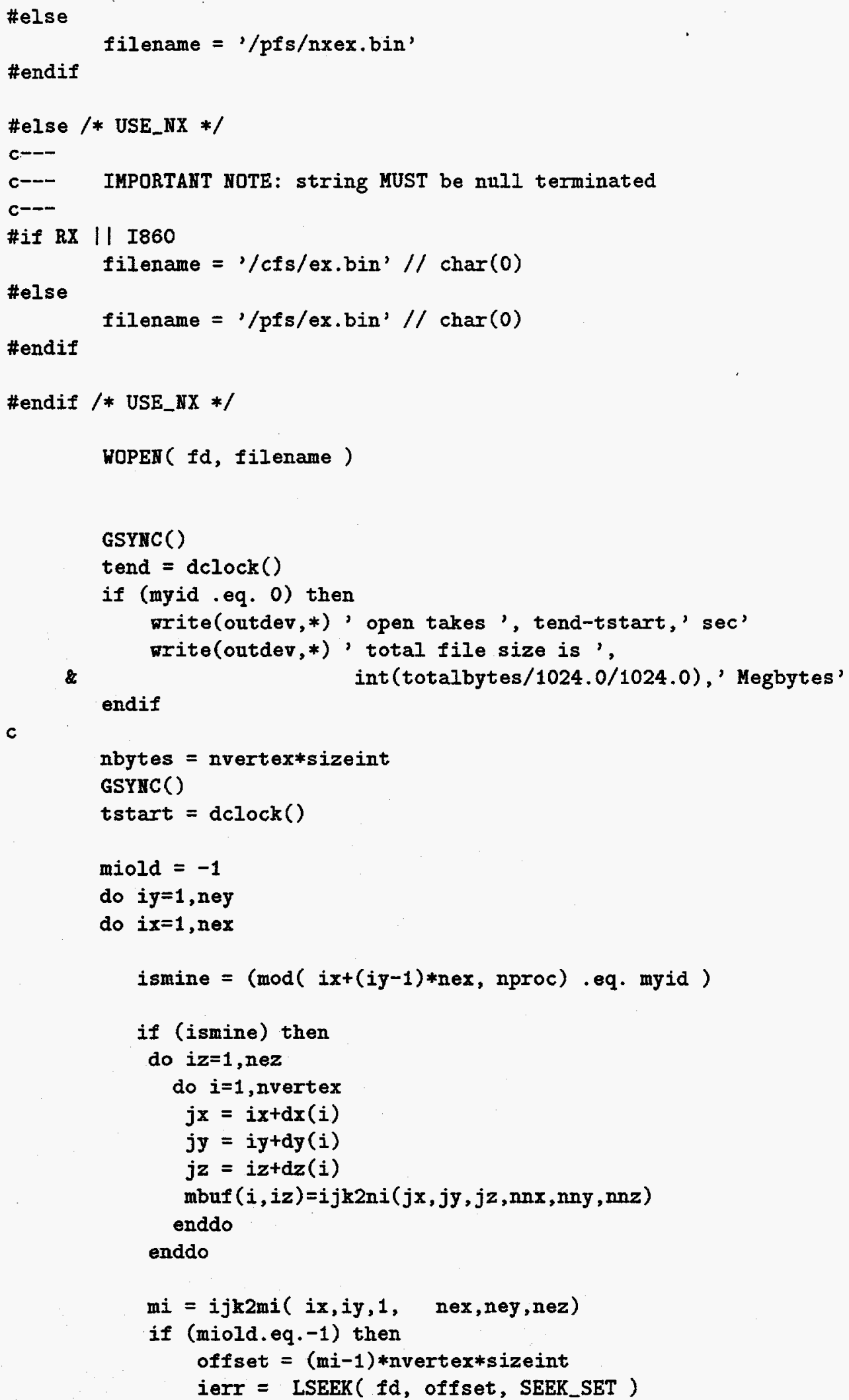




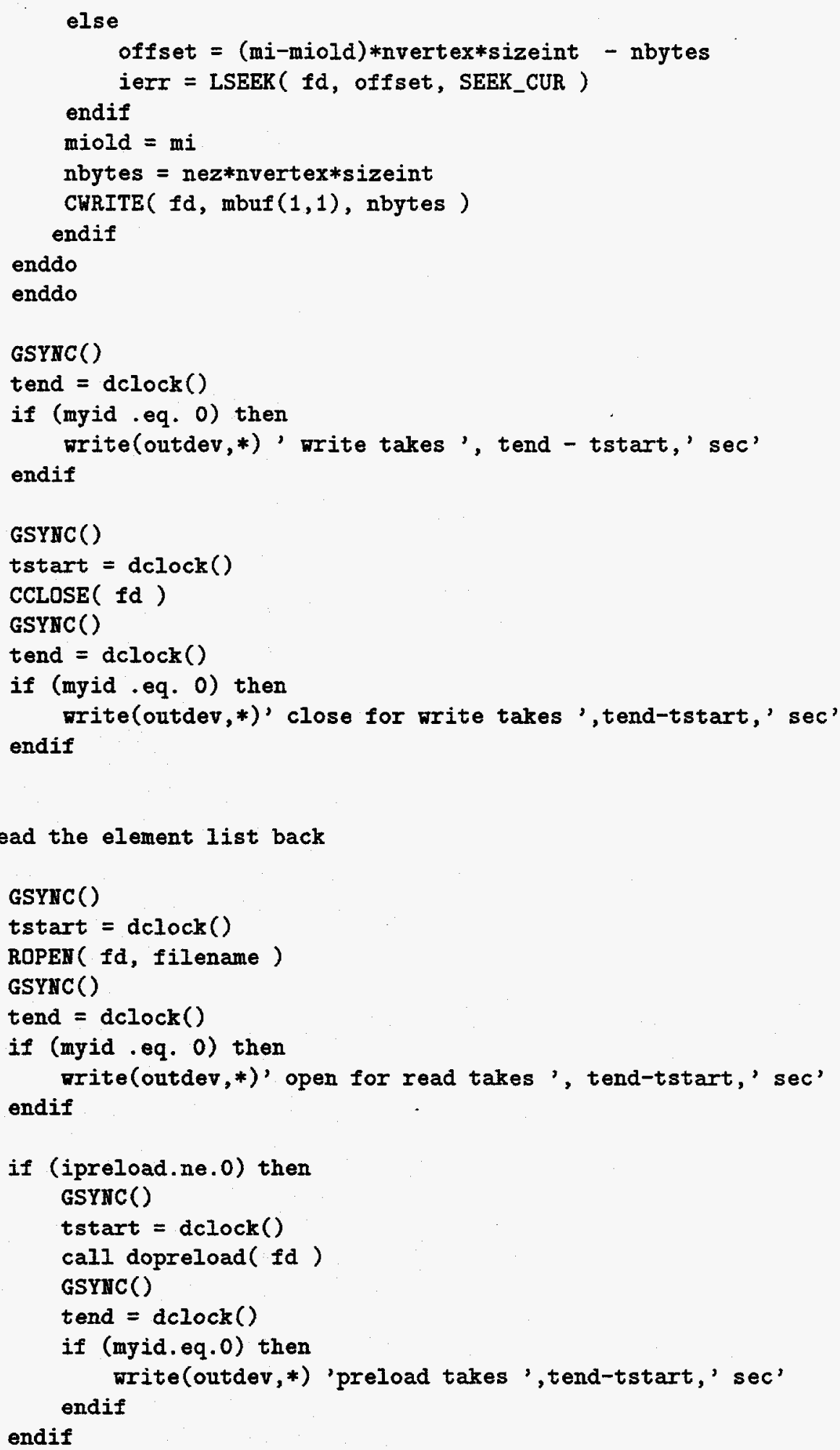




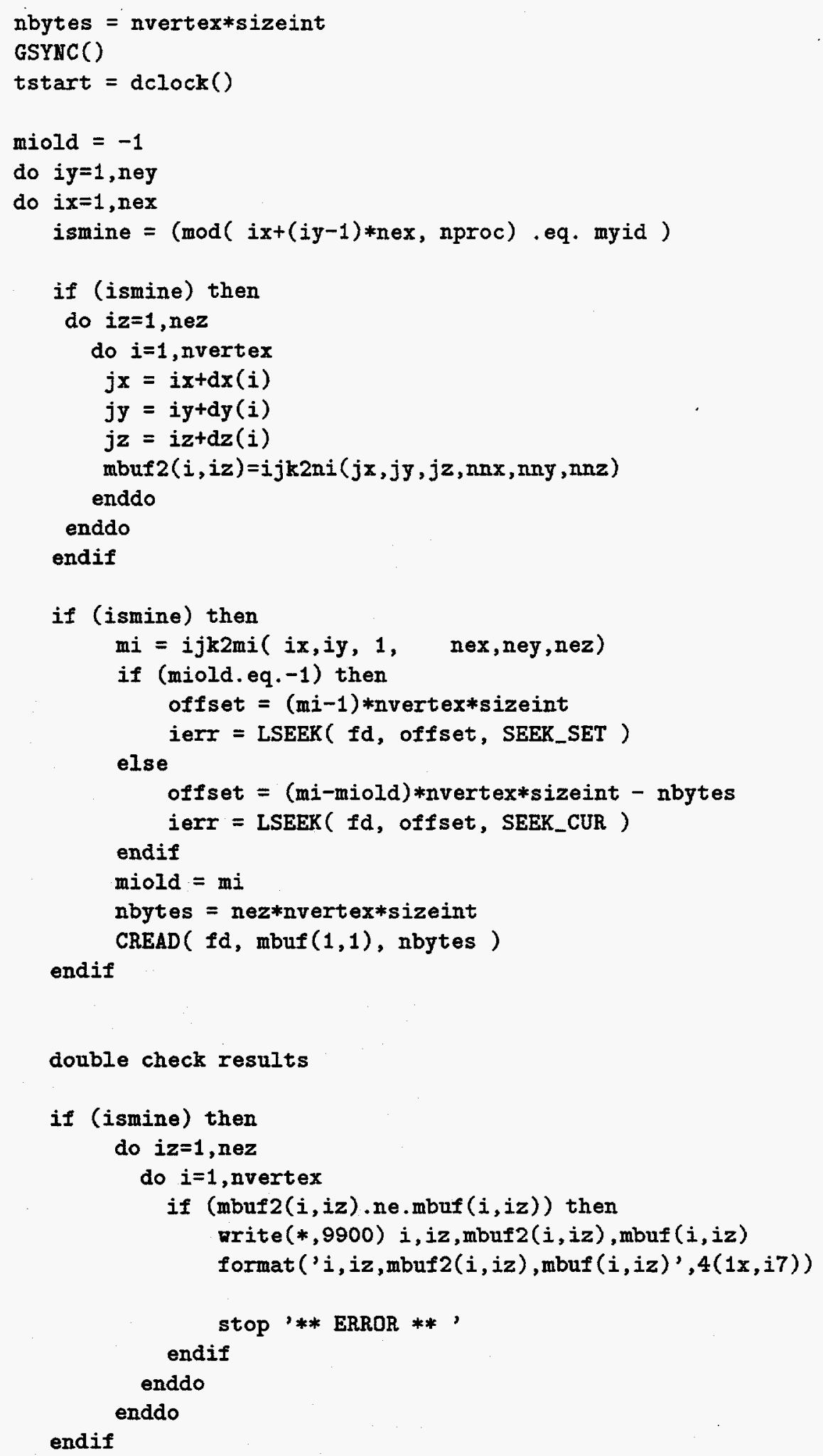




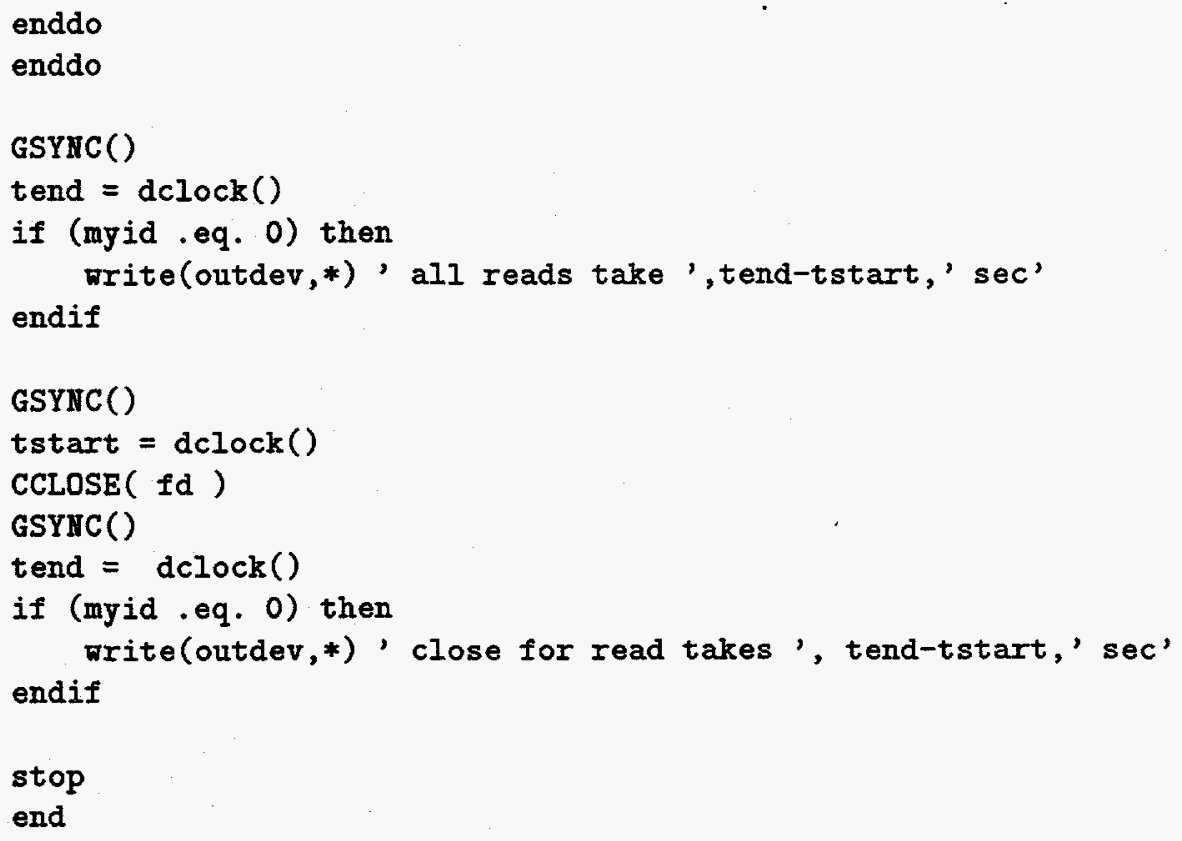




\section{References}

[1] E. F. D'Azevedo and C. H. Romine, DOLIB: Distributed Object Library, Tech. Report ORNL/TM-12744, Oak Ridge National Laboratory, 1994.

[2] — DONIO: Distributed Object Network I/O Library, Tech. Report ORNL/TM12743, Oak Ridge National Laboratory, 1994.

[3] B. Marr, R. Peierls, ANd J. PASciak, IPX - Preemptive remote procedure execution for concurrent applications, Tech. Report, Brookhaven National Laboratory, 1994. 
ORNL/TM-12934

\section{INTERNAL DISTRIEUTION}

1. B. R. Appleton

2. B. A. Carreras

3-7. E. F. D'Azevedo

8. T. S. Darland

9. J. J. Dongarra

10. J. B. Drake

11. T. H. Dunigan

12. W. R. Emanuel

13. G. A. Geist

14. K. L. Kliewer

15-19. M. R. Leuze

20. E. G. $\mathrm{Ng}$

21. C. E. Oliver

22. B. W. Peyton

23-27. S. A. Raby
28. B. A. Riley

29-33. C. H. Romine

34. W. A. Shelton

35-39. R. F. Sincovec

40. G. M. Stocks

41. M. R. Strayer

42. D. W. Walker

43. P. H. Worley

44. T. Zacharia

45. Central Research Library

46. ORNL Patent Office

47. K-25 Applied Technology Library

48. Y-12 Technical Library

49. Laboratory Records - RC

50-51. Laboratory Records Dept.

\section{EXTERNAL DISTRIEUTION}

52. Loyce M. Adams, Applied Mathematics, FS-20, University of Washington, Seattle, WA 98195

53. Christopher R. Anderson, Department of Mathematics, University of California, Los Angeles, CA 90024

54. Todd Arbogast, Department of Mathematical Sciences, Rice University, P. O. Box 1892, Houston, TX 77251

55. Donald M. Austin, 6196 EECS Building, University of Minnesota, 200 Union Street, S.E., Minneapolis, MN 55455

56. Robert G. Babb, Oregon Graduate Center, CSE Department, 19600 N.W. Walker Road, Beaverton, OR 97006

57. David H. Bailey, NASA Ames, Mail Stop 258-5, NASA Ames Research Center, Moffet Field, CA 94035

58. Jesse L. Barlow, Department of Computer Science and Engineering, 220 Pond Laboratory, The Pennsylvania State University, University Park, PA 16802-6106

59. Edward H. Barsis, Computer Science and Mathematics, P. O. Box 5800, Sandia National Laboratory, Albuquerque, NM 87185

60. Adam Beguelin, Carnegie Mellon University, School of Computer Science, 5000 Forbes Avenue, Pittsburgh, PA 15213-3890

61. Robert E. Benner, Parallel Processing Division 1413, Sandia National Laboratories, P. O. Box 5800, Albuquerque, NM 87185 
62. Marsha J. Berger, Courant Institute of Mathematical Sciences, 251 Mercer Street, New York, NY 10012

63. Philippe Berger, Institut National Polytechnique, ENSEEIHT, 2 rue Charles CamichelF, 31071 Toulouse Cedex, France

64. Ake Bjorck, Department of Mathematics, Linkoping University, S-581 83 Linkoping, Sweden

65. John H. Bolstad, L-16, Lawrence Livermore National Laboratory, P. O. Box 808, Livermore, CA 94550

66. Roger W. Brockett, Harvard University, Pierce Hall, 29 Oxford Street Cambridge, MA 02138

67. James C. Browne, Department of Computer Sciences, University of Texas, Austin, TX 78712

68. Bill L. Buzbee, Scientific Computing Division, National Center for Atmospheric Research, P. O. Box 3000, Boulder, CO 80307

69. Donald A. Calahan, Department of Electrical and Computer Engineering, University of Michigan, Ann Arbor, MI 48109

70. Thomas A. Callcott, Director, The Science Alliance Program, 53 Turner House, University of Tennessee, Knoxville, TN 37996

71. Tony Chan, Department of Mathematics, University of California, Los Angeles, 405 Hilgard Avenue, Los Angeles, CA 90024

72. Jagdish Chandra, Army Research Office, P. O. Box 12211, Research Triangle Park, NC 27709

73. Siddhartha Chatterjee, Dept. of Computer Science, CB 3175, Sitterson Hall, The University of North Carolina, Chapel Hill, NC 27599-3175

74. Melvyn Ciment, National Science Foundation, 1800 G Street N.W., Washington, DC 20550

75. Tom Coleman, Department of Computer Science, Cornell University, Ithaca, NY 14853

76. Alva Couch, Department of Computer Science, Tufts University, Medford, MA 02155

77. Paul Concus, Mathematics and Computing, Lawrence Berkeley Laboratory, Berkeley, CA 94720

78. Tom Crockett, ICASE, Mail Stop 132C, NASA Langley Research Center, Hampton, VA 23665-5225

79. Jane K. Cullum, IBM T. J. Watson Research Center, P. O. Box 218, Yorktown Heights, NY 10598

80. George Cybenko, Center for Supercomputing Research and Development, University of Illinois, 104 South Wright Street, Urbana, IL 61801-2932

81. Helen Davis, Computer Science Department, Stanford University, Stanford, CA 94305

82. Michel Dayde, Institut National Polytechnique, ENSEEIHT, 2 rue Charles CamichelF, 31071 Toulouse Cedex, France 
83. Craig Douglas, IBM T. J. Watson Research Center, P. O. Box 218, Yorktown Heights, NY 10598-0218

84. Iain S. Duff, Atlas Centre, Rutherford Appleton Laboratory, Chilton, Oxon OX11 OQX, England

85. Victor Eijkhout, University of Tennessee, 107 Ayres Hall, Department of Computer Science, Knoxville, TN 37996-1301

86. Stanley Eisenstat, Department of Computer Science, Yale University, P. O. Box 2158 Yale Station, New Haven, CT 06520

87. Howard C. Elman, Computer Science Department, University of Maryland, College Park, MD 20742

88. Richard E. Ewing, Director, Institute for Scientific Computations, Texas A\&M University, College Station, TX 77843-3404

89. Edward Felten, Department of Computer Science, University of Washington, Seattle, WA 98195

90. Charles Fineman, Ames Research Center, Mail Stop 269/3, Moffet Field, CA 94035

91. David Fisher, Department of Mathematics, Harvey Mudd College, Claremont, CA 91711

92. Jon Flower, Parasoft Corporation, 2500 E. Foothill Boulevard, Suite 205, Pasadena, CA 91107

93. Geoffrey C. Fox, NPAC, 111 College Place, Syracuse University, Syracuse, NY $13244-4100$

94. Chris Fraley, Statistical Sciences, Inc., 1700 Westlake Avenue N, Suite 500, Seattle, WA 98119

95. Joan M. Francioni, Computer Science Department, University of Southwestern Louisiana, Lafayette, LA 70504

96. Paul O. Frederickson, ACL, MS B287, Los Alamos National Laboratory, Los Alamos, NM 87545

97. Offir Frieder, George Mason University, Science and Technology Building, Computer Science Department, 4400 University Drive, Fairfax, Va 22030-4444

98. Robert E. Funderlic, Department of Computer Science, North Carolina State University, Raleigh, NC 27650

99. Dennis B. Gannon, Computer Science Department, Indiana University, Bloomington, IN 47401

100. C. William Gear, NEC Research Institute, 4 Independence Way, Princeton, NJ 08540

101. W. Morven Gentleman, Division of Electrical Engineering, National Research Council, Building M-50, Room 344, Montreal Road, Ottawa, Ontario, Canada K1A 0R8

102. Alan George, Vice President, Academic and Provost, Needles Hall, University of Waterloo, Waterloo, Ontario, Canada N2L 3G1 
103. James Glimm, SUNY-Stony Brook, Department of Applied Mathematics and Statistics, Stony Brook, NY 11794

104. Gene Golub, Computer Science Department, Stanford University, Stanford, CA 94305

105. Joseph F. Grcar, Division 8245, Sandia National Laboratories, Livermore, CA 94551-0969

106. William D. Gropp, Mathematics and Computer Science Division, Argonne National Laboratory, 9700 South Cass Avenue, Argonne, IL 60439

107. Eric Grosse, AT\&T Bell Labs 2T-504, Murray Hill, NJ 07974

108. Sanjay Gupta, ICASE, Mail Stop 132C, NASA Langley Research Center, Hampton, VA 23665-5225

109. John L. Gustafson, Ames Laboratory, 236 Wilhelm Hall, Iowa State University, Ames, IA 50011-3020

110. Christian Halloy, Assistant Director of JICS, 104 South College, Joint Institute for Computational Science, University of Tennessee, Knoxville, TN 37996-1301

111. Sven J. Hammarling, The Numerical Algorithms Group, Ltd., Wilkinson House, Jordan Hill Road, Oxford OX2 8DR, United Kingdom

112. Robert M. Haralick, Department of Electrical Engineering, Director, Intelligent Systems Lab, University of Washington, 402 Electrical Engineering Building, FT10, Seattle, WA 98195

113. Ann H. Hayes, Computing and Communications Division, Los Alamos National Laboratory, Los Alamos, NM 87545

114. Michael T. Heath, National Center for Supercomputing Applications, 4157 Beckman Institute University of Illinois, 405 North Mathews Avenue, Urbana, IL $61801-2300$

115. Gerald W. Hedstrom, L-71, Lawrence Livermore National Laboratory, P. O. Box 808, Livermore, CA 94550

116. Don E. Heller, Ames Laboratory, 327 Wilhelm, Ames, IA 50011

117. John L. Hennessy, CIS 208, Stanford University, Stanford, CA 94305

118. N. J. Higham, Department of Mathematics, University of Manchester, Gtr Manchester, M13 9PL, England

119. Dan Hitchcock, Office of Scientific Computing, ER-7, Applied Mathematical Sciences, Office of Energy Research, U.S. Department of Energy, Washington, DC 20585

120. Charles J. Holland, Air Force Office of Scientific Research, Building 410, Bolling Air Force Base, Washington, DC 20332

121. Fred Howes, Office of Scientific Computing, ER-7, Applied Mathematical Sciences, Office of Energy Research, Department of Energy, Washington, DC 20585

122. Robert E. Huddleston, Computation Department, Lawrence Livermore National Laboratory, P. O. Box 808, Livermore, CA 94550

123. Jenq-Neng Hwang, Department of Electrical Engineering, FT-10, University of Washington, Seattle, WA 98195 
124. Ilse Ipsen, Department of Computer Science, Yale University, P. O. Box 2158 Yale Station, New Haven, CT 06520

125. Leah H. Jamieson, School of Electrical Engineering, Purdue University, West Lafayette, IN 47907

126. Gary Johnson, Office of Scientific Computing, ER-7, Applied Mathematical Sciences, Office of Energy Research, U.S. Department of Energy, Washington, DC 20585

127. Lennart Johnsson, Thinking Machines Corporation, 245 First Street, Cambridge, MA 02142-1214

128. Harry Jordan, Department of Electrical and Computer Engineering, University of Colorado, Boulder, CO 80309

129. Bo Kagstrom, Institute of Information Processing, University of Umea, 5-901 87 Umea, Sweden

130. Malvyn Kalos, Cornell Theory Center, Engineering and Theory Center Building, Cornell University, Ithaca, NY 14853-3901

131. Hans Kaper, Mathematics and Computer Science Division, Argonne National Laboratory, 9700 South Cass Avenue, Bldg. 221, Argonne, IL 60439

132. Alan H. Karp, HP Labs 3U-7, Hewlett-Packard Company, 1501 Page Mill Road, Palo Alto, CA 94304

133. Linda Kaufman, Bell Laboratories, 600 Mountain Avenue, Murray Hill, NJ 07974

134. Robert J. Kee, Applied Mathematics Division 8245, Sandia National Laboratories, Livermore, CA 94551-0969

135. Kenneth Kennedy, Department of Computer Science, Rice University, P.O. Box 1892, Houston, TX 77001

136. Tom Kitchens, Office of Scientific Computing, ER-7, Applied Mathematical Sciences, Office of Energy Research, U.S. Department of Energy, Washington, DC 20585

137. Clyde P. Kruskal, Department of Computer Science, University of Maryland, College Park, MD 20742

138. Edward Kushner, Intel Corporation, 15201 NW Greenbrier Parkway, Beaverton, OR 97006

139. Michael Langston, Department of Computer Science, University of Tennessee, Knoxville, TN 37996-1301

140. Richard Lau, Office of Naval Research, Code 111MA 800 Quincy Street, Boston Tower 1, Arlington, VA 22217-5000

141. Robert L. Launer, Army Research Office, P. O. Box 12211, Research Triangle Park, NC 27709

142. Tom Leighton, Lab for Computer Science, Massachusetts Institute of Technology, 545 Technology Square, Cambridge, MA 02139

143. Robert Leland, Sandia National Laboratories, 1424, P. O. Box 5800 , Albuquerque, NM 87185-5800 
144. Randall J. LeVeque, Applied Mathematics, FS-20, University of Washington, Seattle, WA 98195

145. John G. Lewis, Boeing Computer Services, P. O. Box 24346, M/S 7L-21, Seattle, WA 98124-0346

146. Heather M. Liddell, Center for Parallel Computing, Department of Computer Science and Statistics, Queen Mary College, University of London, Mile End Road, London E1 4NS, England

147. Brent Lindquist, SUNY-Stony Brook, Department of Applied Mathematics and Statistics, Stony Brook, NY 11794

148. Rik Littlefield, Pacific Northwest Laboratory, MS K1-87, P.O.Box 999, Richland, WA 99352

149. Joseph Liu, Department of Computer Science, York University, 4700 Keele Street, Downsview, Ontario, Canada M3J 1P3

150. Franklin Luk, Department of Computer Science, Amos Eaton Building - No. 131 Rensselaer Polytechnic Institute Troy, NY 12180-3590

151. Ewing Lusk, Mathematics and Computer Science Division, Argonne National Laboratory, 9700 South Cass Avenue, MCS 221 Argonne, IL 60439-4844

152. Allen D. Malony, Department of Computer and Information Science, University of Oregon, Eugene, OR 97403

153. Thomas A. Manteuffel, Department of Mathematics, University of Colorado Denver, Denver, CO 80202

154. Anita Mayo, IBM T. J. Watson Research Center, P. O. Box 218, Yorktown Heights, NY 10598

155. Oliver McBryan, University of Colorado at Boulder, Department of Computer Science, Campus Box 425, Boulder, CO 80309-0425

156. James McGraw, Lawrence Livermore National Laboratory, L-306, P. O. Box 808, Livermore, CA 94550

157. Piyush Mehrotra, ICASE, Mail Stop 132C, NASA Langley Research Center, Hampton, VA 23665

158. Paul C. Messina, Mail Code 158-79, California Institute of Technology, $1201 \mathrm{E}$. California Boulevard, Pasadena, CA 91125

159. Cleve B. Moler, MathWorks, 325 Linfield Place, Menlo Park, CA 94025

160. Jorge J. More, Mathematics and Computer Science Division, Argonne National Laboratory, 9700 South Cass Avenue, Argonne, IL 60439

161. William A. Mulder, Koninklijke Shell Exploratie en Produktie Laboratorium, Postbus 60, 2280 AB Rijswijk, The Netherlands

162. David Nelson, Director, Office of Scientific Computing, ER-7, Applied Mathematical Sciences, Office of Energy Research, U.S. Department of Energy, Washington, DC 20585

163. V. E. Oberacker, Department of Physics, Vanderbilt University, Box 1807, Station B, Nashville, TN 37235 
164. Dianne P. O'Leary, Computer Science Department, University of Maryland, College Park, MD 20742

165. Joseph Oliger, Computer Science Department, Stanford University, Stanford, CA 94305

166. James M. Ortega, Department of Computer Science, Thornton Hall, University of Virginia, Charlottesville, VA 22901

167. Steve Otto, Oregon Graduate Institute, Department of Computer Science and Engineering, $19600 \mathrm{NW}$ von Neumann Drive, Beaverton, OR 97006-1999

168. Cherri Pancake, Department of Computer Science, Oregon State University, Corvallis, OR 97331-3202

169. Joseph E. Pasciak, Applied Mathematics, Brookhaven National Laboratory, Upton, NY 11973

170. Merrell Patrick, National Science Foundation, 1800 G Street N.W., Washington, DC 20550

171. David Payne, Intel Corporation, Supercomputer Systems Division, 15201 NW Greenbrier Parkway, Beaverton, OR 97006

172. Ronald F. Peierls, DAS - Bldg. 490-D, P.O. Box 5000, Brookhaven National Laboratory, Upton, NY 11973

173. Linda R. Petzold, Computer Science Department, University of Minnesota, 200 Union Street, S.E., Room 4-192, Minneapolis, MN 55455

174. Dan Pierce, Boeing Computer Services, P.O. Box 24346, M/S 7L-21, Seattle, WA 98124-0346

175. Paul Pierce, Intel Corporation, Supercomputer Systems Division, 15201 NW Greenbrier Parkway, Beaverton, OR 97006

176. Robert J. Plemmons, Departments of Mathematics and Computer Science, North Carolina State University, Raleigh, NC 27650

177. James C. T. Pool, Deputy Director, Caltech Concurrent Supercomputing Facility, California Institute of Technology, MS 158-79, Pasadena, CA 91125

178. Jesse Poore, Computer Science Department, University of Tennessee, Knoxville, TN 37996-1300

179. David A. Poplawski, Department of Computer Science, Michigan Technological University, Houghton, MI 49931

180. Roldan Pozo, University of Tennessee, 107 Ayres Hall, Department of Computer Science, Knoxville, TN 37996-1301

181. Padma Raghavan, University of Illinois, NCSA, 4151 Beckman Institute, 405 North Matthews Avenue, Urbana, IL 61801

182. Daniel A. Reed, Computer Science Department, University of Illinois, Urbana, IL 61801

183. John K. Reid, Numerical Analysis Group, Central Computing Department, Atlas Centre, Rutherford Appleton Laboratory, Didcot, Oxon OX11 0QX, England

184. John R. Rice, Computer Science Department, Purdue University, West Lafayette, IN 47907 
185. Garry Rodrigue, Numerical Mathematics Group, Lawrence Livermore National Laboratory, Livermore, CA 94550

186. Donald J. Rose, Department of Computer Science, Duke University, Durham, NC 27706

187. Ahmed H. Sameh, Department of Computer Science, University of Minnesota, 200 Union Street S.E., Minneapolis, MN 55455

188. Joel Saltz, Computer Science Department, A.V. Williams Building, University of Maryland, College Park, MD 20742

189. Jorge Sanz, IBM Almaden Research Center, Department K53/802, 650 Harry Road, San Jose, CA 95120

190. Robert B. Schnabel, Department of Computer Science, University of Colorado at Boulder, ECOT 7-7 Engineering Center, Campus Box 430, Boulder, CO 803090430

191. Robert Schreiber, RIACS, MS 230-5, NASA Ames Research Center, Moffet Field, CA 94035

192. Martin H. Schultz, Department of Computer Science, Yale University, P. O. Box 2158 Yale Station, New Haven, CT 06520

193. David S. Scott, Intel Scientific Computers, 15201 N.W. Greenbrier Parkway, Beaverton, OR 97006

194. The Secretary, Department of Computer Science and Statistics, The University of Rhode Island, Kingston, RI 02881

195. Charles L. Seitz, Department of Computer Science, California Institute of Technology, Pasadena, CA 91125

196. Margaret L. Simmons, Computing and Communications Division, Los Alamos National Laboratory, Los Alamos, NM 87545

197. Horst D. Simon, NASA Ames Research Center, Mail Stop T045-1, Moffett Field, CA 94035

198. William C. Skamarock, 3973 Escuela Court, Boulder, CO 80301

199. Tony Skjellum, Dept of Computer Science, Mississippi State University, PO Drawer CS, Mississippi State, MS 39762-5623

200. Burton Smith, Tera Computer Company, 400 North 34th Street, Suite 300, Seattle, WA 98103

201. Marc Snir, IBM T.J. Watson Research Center, Department 420/36-241, P. O. Box 218, Yorktown Heights, NY 10598

202. Larry Snyder, Department of Computer Science and Engineering, FR-35, University of Washington, Seattle, WA 98195

203. Danny C. Sorensen, Department of Mathematical Sciences, Rice University, P. O. Box 1892, Houston, TX 77251

204. Rick Stevens, Mathematics and Computer Science Division, Argonne National Laboratory, 9700 South Cass Avenue, Argonne, IL 60439

205. G. W. Stewart, Computer Science Department, University of Maryland, College Park, MD 20742 
206. Paul N. Swarztrauber, National Center for Atmospheric Research, P. O. Box 3000, Boulder, CO 80307

207. Julie Swisshelm, Sandia National Laboratories, 1421, Parallel Computational Sciences Department, Albuquerque, New Mexico 87185-5800

208. Wei Pai Tang, Department of Computer Science, University of Waterloo, Waterloo, Ontario, Canada N2l 3G1

209. Bernard Tourancheau, LIP ENS-Lyon 69364, Lyon cedex 07, France

210. Joseph F. Traub, Department of Computer Science, Columbia University, New York, NY 10027

211. Lloyd N. Trefethen, Department of Computer Science, Cornell University, Ithaca, NY 14853

212. Robert van de Geijn, University of Texas, Department of Computer Sciences, TAI 2.124, Austin, TX 78712

213. Charles Van Loan, Department of Computer Science, Cornell University, Ithaca, NY 14853

214. Udaya B. Vemulapati, Department of Computer Science, University of Central Florida, Orlando, FL 32816-0362

215. Robert G. Voigt, National Science Foundation, Room 417, 1800 G Street N.W., Washington, DC 20550

216. Bi R. Vona, Center for Numerical Analysis, RLM 13.150, University of Texas at Austin, Austin, TX 78712

217. Henk A. van der Vorst, Professor Dept. of Mathematics, Universiteit Utrecht, P.O. Box 80010, 3508 TA, Utrecht, THE NETHERLANDS

218. Michael D. Vose, 107 Ayres Hall, Department of Computer Science, University of Tennessee, Knoxville, TN 37996-1301

219. Phuong Vu, Cray Research, Inc., 19607 Franz Road, Houston, TX 77084

220. A. J. Wathen, School of Mathematics, University Walk, Bristol BSB 1TW, England

221. Robert P. Weaver, 1555 Rockmont Circle, Boulder, CO 80303

222. Mary F. Wheeler, Department of Mathematical Sciences, Rice University, P. O. Box 1892, Houston, TX 77251

223. Andrew B. White, Computing Division, Los Alamos National Laboratory, Los Alamos, NM 87545

224. John Zahorjan, Department of Computer Science and Engineering, Sieg Hall, FR35, University of Washington, Seattle, WA 98195

225. Office of Assistant Manager for Energy Research and Development, U.S. Department of Energy, Oak Ridge Operations Office, P. O. Box 2001, Oak Ridge, TN $37831-8600$

226-227. Office of Scientific \& Technical Information, P. O. Box 62, Oak Ridge, TN 37831 